Tôhoku Math. Journ.

24 (1972), 51-54.

\title{
POSITIVELY CURVED COMPLEX HYPERSURFACES IMMERSED IN A COMPLEX PROJECTIVE SPACE
}

\author{
KoICHI OGIUE
}

(Received Aug. 16, 1971)

1. Introduction. Let $P_{m}(\boldsymbol{C})$ be a complex projective space of complex dimension $m$ with the Fubini-Study metric of constant holomorphic sectional curvature 1. Recently, using topological methods in algebraic geometry, we have proved the following result.

Proposition A ([2]). Let $M$ be a complete complex hypersurface imbedded in $P_{n+1}(C)$. If $n \geqq 2$ and if every sectional curvature of $M$ with respect to the induced metric is positive, then $M$ is complex analytically isometric to a hyperplane $P_{n}(C)$.

S. Tanno has tried to generalize this result to immersed hypersurfaces and obtained the following.

Proposition B ([4]). Let $M$ be a complete complex hypersurface immersed in $P_{n+1}(C)$. If $n \geqq 2$ and if every sectional curvature of $M$ with respect to the induced metric is greater than $(1 / 4)\{1-(n+2) /(3 n)\}$, then $M$ is complex analytically isometric to a hyperplane $P_{n}(C)$.

The purpose of this paper is to prove the following theorem which is a generalization of Proposition $\mathrm{A}$ and is also an improvement of proposition $\mathrm{B}$ in the case $n \geqq 4$.

THEOREM. Let $M$ be a complete complex hypersurface immersed in $P_{n+1}(C)$. If $n \geqq 4$ and if every sectional curvature of $M$ with respect to the induced metric is positive, then $M$ is complex analytically isometric to a hyperplane $P_{n}(C)$.

2. Proof of Theorem. First we note that since every sectional curvature of $M$ is positive, $M$ is compact (cf., Proposition 3.1 in [2]).

Let $J($ resp. $\widetilde{J})$ be the complex structure of $M\left(\right.$ resp. $\left.P_{n+1}(C)\right)$ and $g$ (resp. $\widetilde{g})$ be the Kaehler metric of $M\left(\operatorname{resp} . P_{n+1}(\boldsymbol{C})\right)$. We denote by $\nabla$ (resp. $\tilde{\nabla})$ the covariant differentiation with respect to $g$ (resp. $\widetilde{g}$ ). Then the second fundamantal form $\sigma$ of the immersion is given by

Work done under partial support by the Sakko-kai Foundation. 


$$
\sigma(X, Y)=\tilde{\nabla}_{X} Y-\nabla_{X} Y
$$

Let $R$ be the curvature tensor field of $M$. Then the equation of Gauss is

$$
\begin{aligned}
g(R(X, Y) Z, W)= & \widetilde{g}(\sigma(X, W), \sigma(Y, Z))-\widetilde{g}(\sigma(X, Z), \sigma(Y, W)) \\
& +\frac{1}{4}\{g(X, W) g(Y, Z)-g(X, Z) g(Y, W) \\
& +g(J X, W) g(J Y, Z)-g(J X, Z) g(J Y, W) \\
& +2 g(X, J Y) g(J Z, W)\} .
\end{aligned}
$$

Let $\xi, \widetilde{J} \xi$ be local fields of orthonormal vectors normal to $M$. If we set

$$
\begin{aligned}
g(A X, Y) & =\widetilde{g}(\sigma(X, Y), \xi), \\
g\left(A^{*} X, Y\right) & =\widetilde{g}(\sigma(X, Y), \widetilde{J} \xi),
\end{aligned}
$$

then $A$ and $A^{*}$ are local fields of symmetric linear transformations. We can easily see that $A^{*}=J A$ and $J A=-A J$ so that, in particular,

$$
\operatorname{tr} A=\operatorname{tr} A^{*}=0 \text {. }
$$

The equation of Gauss can be written in terms of $A$ 's as

$$
\begin{aligned}
g(R(X, Y) Z, W)= & g(A X, W) g(A Y, Z)-g(A X, Z) g(A Y, W) \\
& +g(J A X, W) g(J A Y, Z)-g(J A X, Z) g(J A Y, W) \\
& +\frac{1}{4}\{g(X, W) g(Y, Z)-g(X, Z) g(Y, W) \\
& +g(J X, W) g(J Y, Z)-g(J X, Z) g(J Y, W) \\
& +2 g(X, J Y) g(J Z, W)\}
\end{aligned}
$$

Let $\rho$ be the scalar curvature of $M$. Then we have

$$
\rho=n(n+1)-\|\sigma\|^{2},
$$

where $\|\sigma\|$ is the length of the second fundamental form of the immersion so that $\|\sigma\|^{2}=2 \operatorname{tr} A^{2}$. We can see from (1) that the sectional curvature $K$ of $M$ determined by orthonormal vectors $X$ and $Y$ is given by

$$
\begin{aligned}
K(X, Y)= & \frac{1}{4}\left\{1+3 g(X, J Y)^{2}\right\} \\
& +g(A X, X) g(A Y, Y)-g(A X, Y)^{2} \\
& +g(J A X, X) g(J A Y, Y)-g(J A X, Y)^{2} .
\end{aligned}
$$

At each point $x$ of $M$, we can choose an orthonormal basis $e_{1}, \cdots, e_{n}$, $J e_{1}, \cdots, J e_{n}$ of $T_{x}(M)$ with respect to which the matrix of $A$ is of the form 


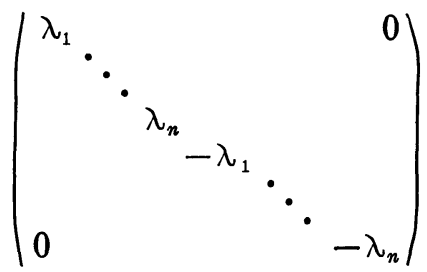

so that

$$
\|\sigma\|^{2}=2 \operatorname{tr} A^{2}=4 \Sigma \lambda_{\alpha}^{2} .
$$

From (3) we have, for $\alpha \neq \beta$,

$$
K\left(\frac{e_{\alpha}+e_{\beta}}{\sqrt{2}}, \frac{J e_{\alpha}-J e_{\beta}}{\sqrt{2}}\right)=\frac{1}{4}-\frac{\lambda_{\alpha}^{2}+\lambda_{\beta}^{2}}{2} .
$$

Since every sectional curvature of $M$ is positive, we have

$$
\lambda_{\alpha}^{2}+\lambda_{\beta}^{2}<\frac{1}{2}
$$

From (4) we have

$$
\lambda_{\alpha}^{4}+\lambda_{\alpha}^{2} \lambda_{\beta}^{2} \leqq \frac{1}{2} \lambda_{\alpha}^{2}
$$

and hence

$$
(n-1) \Sigma \lambda_{\alpha}^{4}+\sum_{\alpha \neq \beta} \lambda_{\alpha}^{2} \lambda_{\beta}^{2} \leqq \frac{n-1}{2} \Sigma \lambda_{\alpha}^{2}
$$

or

$$
(n-2) \Sigma \lambda_{\alpha}^{4}+\left(\Sigma \lambda_{\alpha}^{2}\right)^{2} \leqq \frac{n-1}{2} \Sigma \lambda_{\alpha}^{2}
$$

Therefore we have

$$
(n-2) \operatorname{tr} A^{4}+\frac{1}{2}\left(\operatorname{tr} A^{2}\right)^{2} \leqq \frac{n-1}{2} \operatorname{tr} A^{2},
$$

i.e.,

$$
(n-2) \operatorname{tr} A^{4}+\frac{1}{8}\|\sigma\|^{4} \leqq \frac{n-1}{4}\|\sigma\|^{2} .
$$

On the other hand, we know that the second fundamental form 0 satisfies the following differential equation ([2]).

$$
\frac{1}{2} \Delta\|\sigma\|^{2}=\left\|\nabla^{\prime} \sigma\right\|^{2}-8 \operatorname{tr} A^{4}-\frac{1}{2}\|\sigma\|^{4}+\frac{n+2}{2}\|\sigma\|^{2},
$$

where $\Delta$ denotes the Laplacian and $\nabla^{\prime}$ the covariant differentiation with 
respect to the connection in (tangent bundle) $\bigoplus$ (normal bundle). From (5) we have

$$
-8 \operatorname{tr} A^{4}-\frac{1}{2}\|\sigma\|^{4}+\frac{n+2}{2}\|\sigma\|^{2} \geqq \frac{n-4}{2(n-2)}\|\sigma\|^{2}\left(n-\|\sigma\|^{2}\right) .
$$

Moreover we can see from (4) that $\Sigma \lambda_{\alpha}^{2}<n / 4$, i.e., $\|\sigma\|^{2}<n$. Therefore we have

$$
-8 \operatorname{tr} A^{4}-\frac{1}{2}\|\sigma\|^{4}+\frac{n+2}{2}\|\sigma\|^{2} \geqq 0,
$$

which, together with (6), implies $\Delta\|\sigma\|^{2} \geqq 0$. Hence, by a well-known theorem of E. Hopf, $\|\sigma\|^{2}$ is a constant so that the scalar curvature $\rho$ is a constant. This, combined with Theorem 1 in [1], implies that $M$ is complex analytically isometric to a complex projective space with the Fubini-Study metric of constant holomorphic sectional curvature. Moreover by Theorem 3 in [3] $M$ is imbedded as a totally geodesic hypersurface. This completes the proof of Theorem.

\section{BIBLIOGRAPHY}

[1] R. L. Bishop - S. I. GoldBeRG, On the topology of positively curved Kaehler manifolds II, Tôhoku Math. J., 17 (1965), 310-318.

[2] K. OGIUE, Differential geometry of algebraic manifolds, Differential Geometry, in honor of K. Yano, Kinokuniya, Tokyo (1972), 355-372.

[ 3 ] B. O'NeILL, Isotropic and Kaehler immersions, Canad. J. Math., 17(1965), 907-915.

[4] S. Tanno, Compact complex submanifolds immersed in complex projective spaces, to appear.

Department of Mathematics

TOKyo Metropolitan UnIVersity

TOKYO, JAPAN 